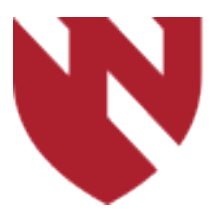

September 2020

\title{
Neurolytic Celiac Plexus Blockade in Patients With Upper Intra- Abdominal Malignancies: An Evidence-Based Narrative Review
}

Apollo Stack

University of Nebraska Medical Center

Kevin Wong

Desert Orthopedic Center

Madhuri Are

University of Nebraska Medical Center

Tell us how you used this information in this short survey.

Follow this and additional works at: https://digitalcommons.unmc.edu/gmerj

Part of the Higher Education Commons, and the Medicine and Health Sciences Commons

\section{Recommended Citation}

Stack, A., Wong, K., , Are, M. Neurolytic Celiac Plexus Blockade in Patients With Upper Intra-Abdominal Malignancies: An Evidence-Based Narrative Review. Graduate Medical Education Research Journal. 2020 Sep 29; 2(1). https://digitalcommons.unmc.edu/gmerj/vol2/iss1/27 


\section{Neurolytic Celiac Plexus Blockade in Patients With Upper Intra-Abdominal Malignancies: An Evidence-Based Narrative Review}

\section{Creative Commons License}

\section{c) (1)@ $\Theta$}

This work is licensed under a Creative Commons Attribution-Noncommercial-No Derivative Works 4.0 License. 


\section{Laboratory Quality Improvement: Utilizing Microsoft Office 365 Collaborative Tools to Improve Communication and Efficiency in Molecular Diagnostic Laboratories \\ Jeff Plambeck', Tareq Qdaisat ${ }^{2}$, Allison Cushman Vokoun ${ }^{2}$}

${ }^{1}$ University of Nebraska Medical Center, Department of Molecular Genetics

${ }^{2}$ University of Nebraska Medical Center, Department of Pathology and Microbiology

Mentor: Allison Cushman Vokoun

Program: Pathology and Microbiology

Type: Original Research

Background: In this day and age, the increased use of traditional communication tools in the work environment such as physical white boards, sticky notes, and emails has become a burden on daily workflow. Issues of specimen tracking, patient privacy, and workflow redundancy are the main concerns. The need for reliable, reproducible, updatable, and efficient laboratory digital whiteboards is becoming obvious, especially with the exponentially increasing amount of molecular genetic testing performed as part of daily clinical practice. The use of commercial systems is limited by high initial investment and maintenance cost, difficulty of implementation, and the lack of ability to be customized.

Methods: We designed an internal laboratory digital whiteboards system using Microsoft planner platform (part of Microsoft (MS) office 360 ). The participants are able to access the platform through desktop computers and secure mobile device applications. Two main plans were created in MS planner: 1) Molecular Diagnostic Team plan; and 2) Heme Team plan. Each plan was divided into buckets (e.g. pending cases, to do list, clinical runs by date, current lab issues, quality control, improvements, and individual molecular pathologist's assigned cases progress). During the transition phase, the implementation of the new system was assessed and edited daily during the laboratory daily huddle. Afterwards, to evaluate the impact of digital whiteboards on the laboratory workflow, quantitative assessment was performed using an online survey questionnaire.

Results: An online survey was developed and sent to the participants who used the platform over a period of four months. In total, $58.3 \%$ responded by the time of collecting the survey responses. All of the respondents did use Microsoft planner for laboratory daily workflow: $100 \%$ used it daily, $100 \%$ thought it was useful to switch to a digital version of whiteboard, $100 \%$ prefered using the platform over the conventional methods, $100 \%$ thought that the new platform improved communication between lab personnel, $100 \%$ thought that switching to the new platform improved workflow efficiency, 71\% thought that switching to the new platform improved communication with pathologists and trainees, $71 \%$ thought that switching to the new platform reduced workflow errors, $57 \%$ thought that switching reduced the time spent tracking cases.

Conclusion: Switching daily molecular laboratory case annotation and tracking system to a digital format may improve staff communication, molecular laboratory workflow, and streamline specimens tracking process.

https://doi.org/10.32873/unmc.dc.gmerj.2.1.025

\section{Neurolytic Celiac Plexus Blockade in Patients With Upper Intra-Abdominal Malignancies: An Evidence- Based Narrative Review Kevin Wong ${ }^{1}$, Apollo Stack ${ }^{2}$, Madhuri Are ${ }^{2}$ \\ ${ }^{1}$ Desert Orthopedic Center, Palm Springs, CA \\ UUniversity of Nebraska Medical Center, Department of Anesthesiology}

Mentor: Madhuri Are

Program: Anesthesiology

Type: Review/Meta-Analysis

Background: Cancer-related abdominal pain is a common symptom associated with upper intra-abdominal carcinoma, especially in patients with advanced disease and it has posed a significant therapeutic challenge to medical practitioners. Typically, cancer pain can be managed by following the World Health Organization 3-step analgesic ladder. However, analgesic use of opioids, the mainstay treatment for moderate-to-severe cancer-related pain, may be ineffective in a subset of cancer patients. Escalation of dosage may be limited by opioid-induced side effects. Thus, with $70-80 \%$ of advance-staged patients, and up to $90 \%$ of those with bone metastases, suffering from pain, ablative procedures including neurolysis of the celiac/splanchnic plexus should be sought to improve analgesic outcomes (1). Our objective was to review the literature addressing the effect of neurolytic celiac plexus block (NCPB) on the palliation of pain emanating from advanced upper intraabdominal malignancies.

Methods: Electronic databases including Medline/PubMed, EMBASE, and Cochrane Library were searched. Only studies with a high level of evidence were reviewed. These included prospective randomized control studies, systematic reviews and meta-analyses. Further, references from included articles were carefully reviewed for additional relevant trials.

Results: A total of 13 prospective randomized trials, five systematic reviews and metaanalyses, and one Cochrane review article were found to meet eligibility criteria.

Conclusion: Neurolysis of the celiac/ splanchnic plexus is an effective and safe therapeutic modality that should be considered early for palliation of cancerrelated pain in advanced upper intraabdominal malignancies. This is especially true for patients with intolerable opioidinduced adverse events and painful symptoms resistant to oral analgesics.

https://doi.org/10.32873/unmc.dc.gmerj.2.1.026 